\title{
L'IMPATTO DEI CAMBIAMENTI CLIMATICI SULLA CRESCITA ECONOMICA E LA DISUGUAGLIANZA GLOBALE ${ }^{1}$
}

\author{
MASSIMO TAVONI (*) \\ Nota presentata dal m.e. Marino Gatto \\ (Adunanza del 29 novembre 2018)
}

SuNTO. - I cambiamenti climatici avranno un impatto sulla crescita economica e aggraveranno le disuguaglianze globali. Colpendo di più i più poveri, sia in Italia, Europa e in tutto il mondo, il riscaldamento globale rappresenta una sfida per l'ordine internazionale. Qui discutiamo brevemente le ultime analisi degli impatti economici dei cambiamenti climatici.

$$
* * *
$$

ABSTRACT. - Climate change will impact economic growth and exacerbate global income inequalities. By hitting the poorest more, both in Italy, Europe and worldwide, it poses a challenge for the international order. Here we discuss the latest analysis of the economic impacts of climate change.

L'impatto dei cambiamenti climatici sulla nostra società sarà molto profondo. Già ad oggi, con un riscaldamento del pianeta di circa 1 grado centigrado, gli impatti sulla disuguaglianza globale si fanno sentire. Ma questo è niente rispetto a quello che avverrà nei prossimi

(i) Professore di Economia dei cambiamenti climatici, Politecnico di Milano; Direttore del RFF CMCC European Institute on Economics and the Environment, Italy. E-mail: massimo.tavoni@polimi.it

1 Sintesi dell'intervento. 
decenni. Il riscaldamento globale supererà i 2 gradi a metà secolo e a seconda delle nostre azioni potrà andare ben oltre. Questo avrà ripercussioni sia sugli ecosistemi - come l'estinzione di ecosistemi e speciesia sull'uomo - su fattori di produzione come lavoro, capitale e risorse naturali. Gli economisti del clima hanno affrontato questo problema ormai da diversi anni, ma ad oggi le stime degli impatti economici rimangono un argomento di ricerca molto fertile e ancora lungi dall'essere consolidate.

Recentemente sono stati sviluppati metodi alternativi per stimare l'impatto economico del clima a partire da dati empirici storici. Questo approccio analizza il modo in cui i cambiamenti di temperatura negli ultimi 40 anni hanno influenzato la crescita economica di tutti i paesi del mondo, tenendo conto delle loro differenze istituzionali, tecnologiche e climatiche. Questa valutazione retrospettiva ha rivelato una relazione non lineare tra temperatura e crescita economica: per i paesi freddi (al di sotto di una temperatura "ideale"), un aumento delle temperature potrebbe favorire l'economia e portare a una crescita aggiuntiva. Per i paesi caldi, tuttavia, porterebbe a una crescita economica ridotta, più significativo è il paese più caldo.

Applicando queste stime a diversi scenari di riscaldamento futuri, si ottengono perdite economiche estremamente significative. Ad esempio, per gli aumenti della temperatura globale di $3^{\circ} \mathrm{C}$, un risultato molto probabile dati gli attuali andamenti delle emissioni, sono state stimate perdite del PIL mondiale tra il 15 e il 60\%. È importante tenere presente che questi studi, poiché estrapolano le informazioni del passato in un futuro con un clima diverso, non includono fattori come l'innalzamento del mare, l'acidificazione degli oceani, ecc., Fattori che potrebbero aumentare principalmente i danni economici al clima. Al contrario, una maggiore adattabilità potrebbe limitare i danni. L'ultimo rapporto IPCC su $1,5^{\circ} \mathrm{C}$ ha mostrato che limitare il riscaldamento a $1,5^{\circ} \mathrm{C}$ invece di $2^{\circ} \mathrm{C}$ consentirebbe di risparmiare 1 '1,5-2,0\% del prodotto lordo mondiale (PIL) di mezzo secolo e il 3,5\% del PIL entro la fine del secolo. Sulla base di un tasso di sconto del $3 \%$, ciò corrisponde a $\$$ 8,1-11,6 trilioni e $\$ 38,5$ trilioni di danni evitati rispettivamente alla metà e alla fine del secolo.

Queste analisi empiriche mostrano come le perdite economiche dovute al cambiamento climatico saranno concentrate principalmente nei paesi caldi, dove il riscaldamento aggiuntivo porta a un forte declino economico. I paesi caldi sono anche più poveri di quelli freddi. Di 
conseguenza, il cambiamento climatico non solo rallenterà la crescita economica globale, ma aggraverà anche le disuguaglianze globali e colpirà più duramente quei paesi che, di fatto, hanno contribuito meno ai cambiamenti climatici causati dall'uomo.

Questo impatto diseguale e regressivo del cambiamento climatico è previsto avvenire anche in Italia, come si nota dalla Fig. 1. Usando metodi statistici simili a quelli descritti sopra, abbiamo stimato che nella seconda metà del secolo la perdita di PIL italiano dovuto al riscaldamento globale potrebbe essere superiore al $8 \%$. In aggiunta, I nostri calcoli suggeriscono un aumento della disuguaglianza regionale in Italia del $60 \%$ nella seconda metà del secolo.

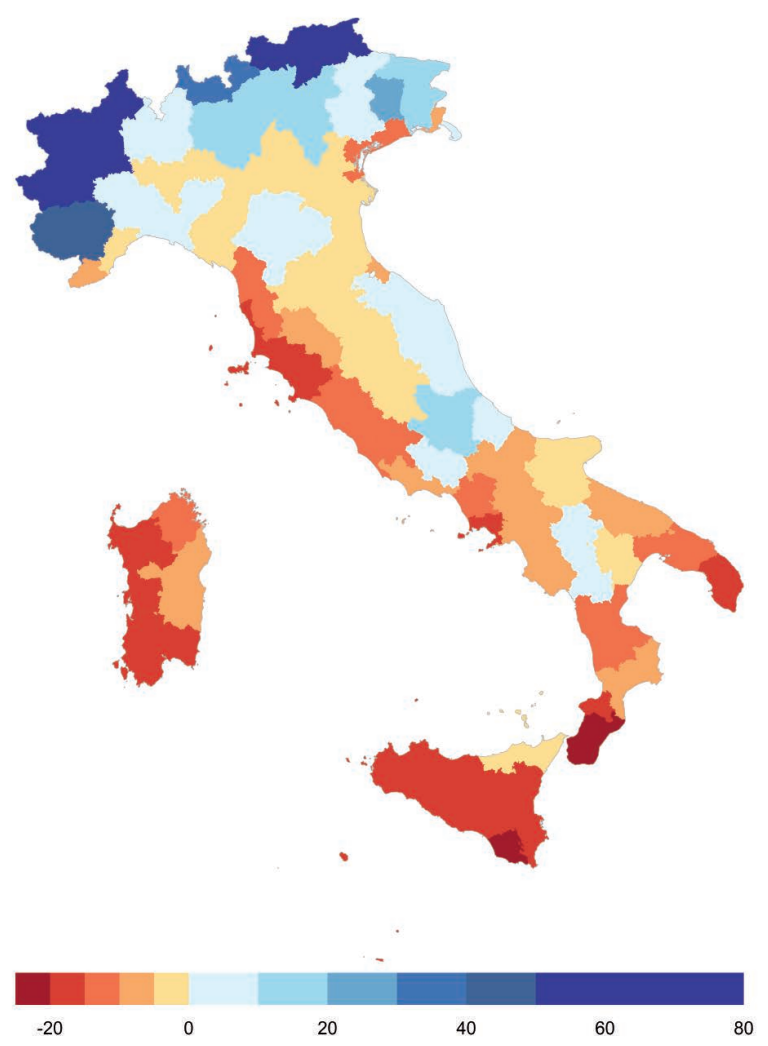

Fig. 1. Impatto del cambiamento climatico sulla crescita economica regionale in Italia. Fonte: 'Impatti economici dei cambiamenti climatici in Italia 'Rapporto per la Fondazione Sviluppo Sostenibile a cura di Massimo Tavoni, Francesco Bosello e Shouro Dasgupta. 
L'aumentata iniquità causata dal cambiamento climatico si aggiunge ai trend di concentrazione della ricchezza che sono una delle caratteristiche fondamentali dello sviluppo economico degli ultimi decenni. Per l'Italia, il divario economico storico fra Nord e Sud del paese rischierebbe di allargarsi ulteriormente. E lo stesso ragionamento vale per il Nord e il Sud dell'Europa, e più in generale del mondo. Risolvere il problema del clima, dunque, significherebbe non solo evitare disastri ecosistemici ed economici, ma anche evitare che le disuguaglianze sociali - con tutte le loro ripercussioni politiche - aumentino sproporzionatamente. 